\section{Die uni- und bilaterale Lähmung des Nervus laryngeus inferior (recurrens)}

C. Sittel ${ }^{1}$, K. Wassermann ${ }^{2}$, F. Mathen ${ }^{2}$, H. E. Eckel ${ }^{3}$

${ }^{1}$ Klinik und Poliklinik für Hals-, Nasen- und Ohrenheilkunde der Universität des Saarlandes

2 Medizinische Klinik III der Universität zu Köln

${ }^{3}$ Klinik und Poliklinik für Hals-, Nasen- und Ohrenheilkunde der Universität zu Köln
Zusammenfassung: Die unilaterale Parese eines Nervus recurrens führt zu einem unvollständigen Glottisschluss und damit zu einer empfindlichen Einbuße der Stimmleistungsfähigkeit. Eine bilaterale Lähmung führt zu einer glottischen Stenose, als klinisches Leitsymptom imponiert der inspiratorische Stridor. Die meisten dieser Läsionen sind iatrogen verursacht, insbesondere duch chirurgische Eingriffe an der Schilddrüse. Aber auch eine Vielzahl anderer Ursachen kommt in Betracht. Ist die Ätiologie unklar, muss eine gründliche diagnostische Abklärung erfolgen. Die indirekte Laryngoskopie bestätigt die Verdachtsdiagnose. Für die weitere Diagnostik kommt der laryngealen Elektromyographie besondere Bedeutung zu. Sie erlaubt die Abgrenzung zur Ankylose im Cricoarytaenoidgelenk und gestattet in vielen Fällen eine zuverlässige Prognose der Ausheilungswahrscheinlichkeit. Während ungünstige Verläufe mit hoher Sicherheit vorhergesagt werden können, ist die komplette Ausheilung jedoch schwierig prognostizierbar. Die Behandlung der unilateralen Rekurrensparese ist überwiegend konservativ logopädisch. Im Fall eines persistierenden glottischen Restspalts kann eine Medialisierung der paretischen Stimmlippe zur Stimmverbesserung erfolgen. Dies ist sowohl über einen offenen Zugang als auch mikrolaryngoskopisch möglich. Bilaterale, dauerhafte Paresen erfordern fast immer eine operative Glottiserweiterung, die heute vorzugsweise mikrolaryngoskopisch erfolgt. Gegenüber einer dauerhaften Tracheotomie ist diesem Verfahren wegen der wesentlich geringeren Beeinträchtigung des Patienten stets der Vorzug zu geben. Die Normalisierung der Atmung wird in der Regel durch eine gewisse Stimmverschlechterung erkauft, die aber bei modernen Verfahren tolerabel bleibt.

Unilateral and Bilateral Recurrent Nerve Paralysis: Unilateral recurrent nerve paralysis leads to glottic insufficiency, causing a significant lack of vocal ability. In contrast, bilateral palsies present with stridor on inspiration due to glottic stenosis. Most of the underlying lesions are iatrogenic, with thyroid surgery being the single most important causative factor. However, a variety of different reasons can lead to such a condition. Whenever aetiology is uncertain a complete diagnostic workup is mandatory. Indirect laryngoscopy confirms the diagnosis. Laryngeal electromyography is of great value because it differentiates between paralysis and ankylosis of the cricoarytaenoid joint. Moreover, in many cases laryngeal electromyogra-

Pneumologie 2001; 55: 568-578

(c) Georg Thieme Verlag Stuttgart · New York ISSN 0934-8387 phy provides a reliable prognosis of clinical outcome. While unfavorable results can be predicted with high accuracy, correct prognosis of complete recovery is more difficult. Speech therapy is the treatment of choice in case of unilateral recurrent nerve palsy. Only if a sigificant glottal gap persists medialization procedures may become useful for voice improvement. Endoscopic as well as open approaches are available for this purpose. Bilateral recurrent nerve palsies need to be addressed surgically in the vast majority of cases. Today, a variety of endoscopic techniques for widening the glottic airway are available. Compared to permanent tracheostomy these procedures have much less impact on the patient's quality of life and should be preferred whenever possible. Inevitably, voice quality is traded for airway normalisation. However, modern surgical techniques accomplish very tolerable phonatory results.

\section{Einleitung}

Das hervorstechende Symptom der plötzlichen, einseitigen peripheren Lähmung des Nervus recurrens ist typischerweise die heisere und verhauchte Stimme. Die unvollständige Adduktion der Stimmlippen verursacht einen ständigen glottischen Restspalt, der zu einem raschen und irregulären Luftverlust führt („wilde Luft“) und eine koordinierte Schwingung der Stimmlippen unmöglich macht. Die Kommunikationsfähigkeit der betroffenen Patienten kann dadurch massiv beeinträchtigt sein. Kommt es nicht zu einer funktionell ausreichenden Erholung der Nervenfunktion, kann eine chirurgische Stimmrehabilitation sinnvoll sein. Verschiedene operative Verfahren stehen zur Verfügung, um den glottischen Restspalt bei Phonation dauerhaft $\mathrm{zu}$ verringern, ohne den Atemweg zu beeinträchtigen.

Die simultane Lähmung beider Nervi recurrentes führt hingegen in erster Linie zu einem inspiratorischen Stridor, dem typischen Leitsymptom jeder extrathorakalen Stenosierung der zentralen Atemwege. Die Atmung kann dabei so stark beeinträchtigt werden, dass eine notfallmäßige Intubation oder Tracheotomie notwendig wird. Die Stimmfunktion ist hingegen weniger gestört, zumindest in der mittleren Sprechstimmlage und bei anstrengungsloser Stimmlautstärke. Häufig wird bei langfristig fortbestehender Lähmung eine chirurgische Intervention zur Erweiterung des glottischen Atemwegs erforderlich, um die Folgen der hochgradigen Atemwegsstenosierung zu lindern. Allerdings sind glottiserweiternde Eingriffe bei der beidseitigen Rekurrenslähmung mit einer Beeinträchtigung der stimmlichen Leistungsfähigkeit 
verbunden, so dass Operateur und Patient stets gemeinsam nach einem akzeptablen Kompromiss bezüglich Atmung und Stimme suchen müssen.

\section{Ätiologie}

Durch seinen langen Verlauf von der Schädelbasis zum Mediastinum und zurück zum Kehlkopf ist der Nervus recurrens besonders gefährdet, durch Traumata oder durch Tumorarrosion in Mitleidenschaft gezogen zu werden. Wenn trotz sorgfältiger und ausgiebiger Differenzialdiagnostik keine Ursache gefunden werden kann, wird die Lähmung als idiopathisch bezeichnet. Die Mehrzahl aller Läsionen des Nervus recurrens ist allerdings iatrogen verursacht [1]. Eine Vielzahl operativer Maßnahmen ist mit einem unterschiedlich großen Risiko einer Rekurrensparese verbunden [2,3]. Dazu gehören in erster Linie Eingriffe an der Schilddrüse, zunehmend aber auch Operationen der Halswirbelsäule über einen anterioren Zugang. Weiterhin können gefäßchirurgische Interventionen am Aortenbogen oder an der Arteria carotis sowie die Chirurgie des Ösophagus, des Halses und der Schädelbasis die Integrität des Nervus recurrens beschädigen. Tab. 1 gibt einen Überblick über die relative Häufigkeit einzelner ätiologischer Faktoren im eigenen Patientengut, wobei eine deutliche Geschlechtsdifferenz zu beobachten ist.

Tab. 1 Ätiologie der Rekurrensparesen, getrennt nach Geschlecht.

\begin{tabular}{llll}
\hline & männlich & weiblich & gesamt \\
\hline idiopathisch & $19 \%$ & $18 \%$ & $18 \%$ \\
Schilddrüsen-Chirurgie & $31 \%$ & $71 \%$ & $54 \%$ \\
zervikale Gefäßchirurgie & $16 \%$ & $0 \%$ & $7 \%$ \\
Halswirbelsäulen-Chirurgie & $8 \%$ & $4 \%$ & $6 \%$ \\
Hals-Chirurgie & $7 \%$ & $0 \%$ & $3 \%$ \\
Thorax-Chirurgie & $14 \%$ & $3 \%$ & $8 \%$ \\
Schädelbasis-Chirurgie & $5 \%$ & $4 \%$ & $4 \%$ \\
gesamt & $100 \%$ & $100 \%$ & $100 \%$ \\
\hline
\end{tabular}

Beidseitige Rekurrenslähmungen werden ganz überwiegend durch Operationen an der Schilddrüse, weniger häufig im Mediastinum, verursacht. Das Risiko der Verwirklichung dieser Komplikation wird bei Erstoperationen an der Schilddrüse mit deutlich unter $1 \%$ angegeben $[4,5]$. Bei Rezidivoperationen und bei Operationen wegen bösartiger Schilddrüsenerkrankungen ist das Risiko einer beidseitigen Rekurrenslähmung deutlich erhöht [6,7]. Die sorgfältige Präparation des Nerven bei der Schilddrüsenoperation und ein intraoperatives neurophysiologisches Monitoring des Nervus recurrens sollen das Risiko von Rekurrensparesen im Zusammenhang mit Schilddrüsenoperationen vermindern helfen $[6,8,9]$. Vollständige Sicherheit bezüglich der Schonung des Nervus recurrens gibt es allerdings in der SchilddrüsenChirurgie offenbar weiterhin nicht.

In der Pneumologie ist ein beidseitiger Stimmlippenstillstand praktisch immer ein Zeichen eines ausgedehnten mediastinalen Tumorbefalls bei inoperablem Bronchialkarzinom oder eines infiltrativ wachsenden Adeno-/Plattenepithelkarzinoms des Ösophagus. Hin und wieder geht der beidseitigen die einseitige Rekurrensparese voraus. Sie ist atemmechanisch zwar folgenlos, erfordert aber als signum mali engmaschige Lungenfunktionskontrollen, damit die rekonstruktive Intervention (Tracheotomie, Chordektomie [10]) rechtzeitig erfolgen kann.

Da die präsentierenden Symptome (Stridor, hochgradige Dyspnoe, Erstickungsangst) zunächst auch an eine maligne subglottische Stenose durch Tumorkompression oder -invasion denken lassen, ist für den untersuchenden Bronchologen die gründliche Funktionsbeurteilung der Stimmlippenmotorik vor Intubation der Trachea obligatorisch.

Je nach dem Grad der Sedierung können die Spontanexkursionen der Stimmlippen nämlich so weit eingeschränkt sein, dass zwischen Parese und situativer Bewegungsminderung nur zu unterscheiden ist, wenn der Patient energisch aufgefordert wird, tief einzuatmen oder zu husten. In Einzelfällen wurden beidseitige Rekurrensparesen mit zusätzlich bestehender therapiebedürftiger maligner Trachealstenose beschrieben [11].

Eine sporadisch auftretende aktive inspiratorische, gelegentlich auch exspiratorische, Adduktionsbewegung der Stimmlippen ist die so genannte „vocal cord dysfunction“. Hierbei imitiert die normal innervierte und ansonsten funktionsfähige Glottis eine beidseitige Rekurrensparese. Es kommt zu heftigen Atemnotattacken. Differenzialdiagnostisch interessant ist die nicht seltene Koexistenz mit einem echten Asthma bronchiale [12].

\section{Diagnostik}

Besteht der Verdacht auf eine ein- oder beidseitige Läsion des Nervus recurrens muss zuerst die Beweglichkeit der Stimmlippen verifiziert werden. Dies erfolgt vorzugsweise durch die indirekte Laryngoskopie mit einem starren $90^{\circ}$-WinkeloptikEndoskop (Abb.1), die jedem HNO-Arzt am wachen Patienten möglich ist. Die Beurteilung der Stimmlippen-Mobilität im Rahmen der flexiblen Bronchoskopie ist im Vergleich dazu wesentlich anfälliger für Fehlbeurteilungen.

Die Stellung der Stimmlippen wird klassifiziert in median (Abb. 2), paramedian (Abb.3) und intermediär (Abb.4), weiterhin sollte darauf geachtet werden, ob der Arytaenoidknor-

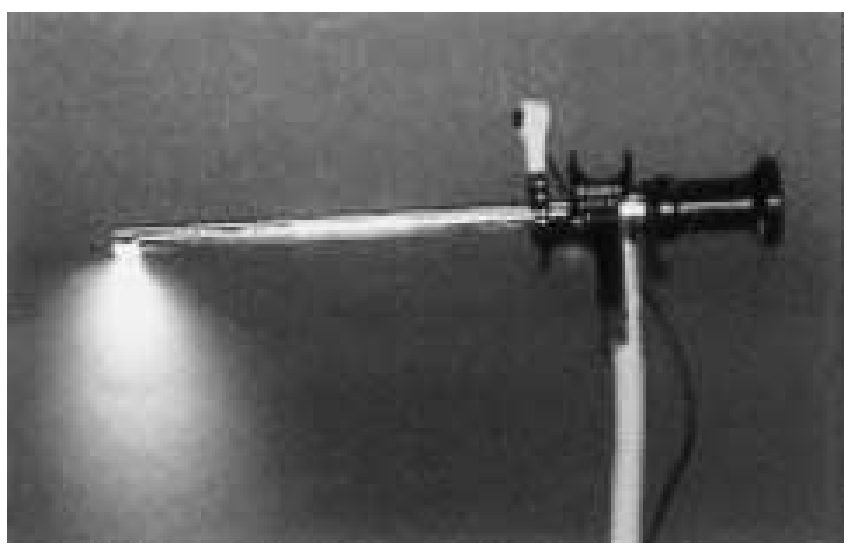

Abb. 1 Starre $90^{\circ}$-Winkeloptik (Lupenlaryngoskop). 


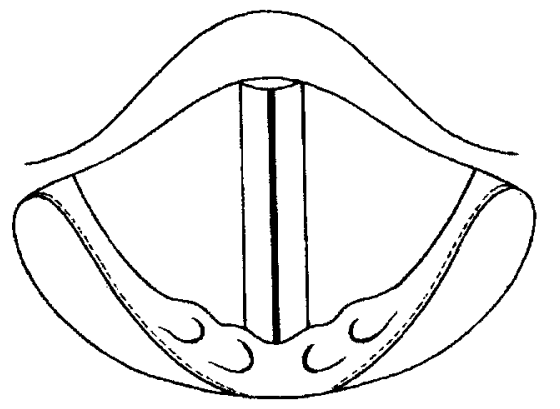

Abb. 2 Glottis schematisch, Stimmlippen median.

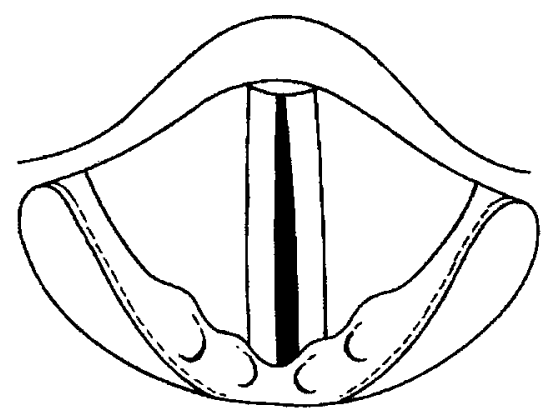

Abb. 3 Glottis schematisch, Stimmlippe rechts paramedian.

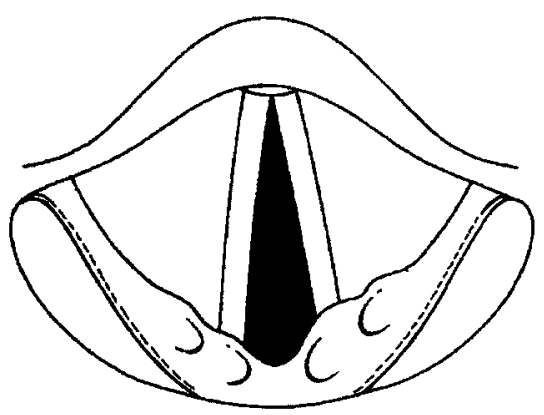

Abb. 4 Glottis schematisch, Stimmlippe rechts intermediär.

pel rotiert und nach medial eingekippt ist. Eine Niveaudifferenz der Stimmlippen ist bei einer beidseitigen Affektion noch schwieriger als ohnehin schon zu verifizieren, kann aber funktionell bedeutsam sein. Falls eine Restbeweglichkeit beobachtet werden kann, ist dies als prognostisch günstiges Zeichen zu werten. Eine Verwechslung mit supraglottischen Kontraktionen ist dabei jedoch recht leicht möglich. Weitere Schlüsse, die über die ad hoc-Bestätigung der Verdachtsdiagnose hinausgehen, sollten aus dieser ersten endoskopischen Untersuchung nicht gezogen werden. Zwar findet sich bis heute in zahlreichen Lehrbüchern das Konzept, dass die Stimmlippenstellung Informationen zum wahrscheinlichen Schädigungsort liefert. Darin spiegelt sich die plausible Vorstellung wider, dass der M. cricothyroideus als einziger vom N. laryngeus superior versorgter Kehlkopfmuskel die Stellung der Stimmlippen in wesentlicher Weise beeinflusst. Eine isolierte Lähmung des $\mathrm{N}$. recurrens soll daher $\mathrm{zu}$ einer anderen Form der Stimmlippenfehlstellung führen als eine isolierte Affektion des N. laryngeus superior oder als eine Kombination beider Lähmungen. Dieses physiologisch und anatomisch naheliegende Modell einer einfachen Topodiagnostik ist jedoch mittlerweile widerlegt worden [13]. Prognostisch verlässliche Hinweise lassen sich aus der einfachen Endoskopie nur sehr eingeschränkt gewinnen: Eine zuverlässig beobachtete Restbeweglichkeit darf sicher als prognostisch günstiges Zeichen gewertet werden. Im Gegensatz dazu ist die beginnende Exkavierung der Stimmlippe als Zeichen der bleibenden Schädigung anzusehen. Dieses typische Symptom der hochgradigen Denervierung tritt jedoch frühestens nach mehreren Wochen auf und kann damit nur sehr eingeschränkt als prognostisches Merkmal bezeichnet werden.

\section{Differenzialdiagnose}

In aller Regel geht der plötzlich einsetzenden Lähmung des N. recurrens ein Ereignis voraus, das typischerweise zu einer solchen Schädigung führen kann, besonders häufig ein operativer Eingriff. Die Anamneseerhebung ist daher in der Regel wegweisend für die ätiologische Eingruppierung der Lähmung. Als wichtige Differenzialdiagnose bleibt aber auch bei vermeintlich klarer Ursache die Ankylose im Cricoarytaenoidgelenk (Abb.5) auszuschließen [14,15]. Ankylosen der Stellknorpel oder Fibrosen der bindegewebigen Kapsel des Cricoarytaenoidgelenks entwickeln sich am häufigsten als Folge vorangegangener Intubationen. Da aber die meisten chirurgischen Eingriffe, die zu einer Läsion des Nervus recurrens führen können, ebenfalls in Intubationsnarkose durchgeführt werden, ist es nicht möglich, alleine von der Anamnese her zwischen mechanisch bedingter Immobilität auf dem Boden einer Ankylose und neurogener Lähmung zu unterscheiden. Zur Differenzierung zwischen mechanischer Bewegungsstörung im Gelenk und neurogener Parese eignet sich einerseits die Elektromyographie des Kehlkopfs [16], andererseits die Prüfung der passiven Mobilität des Stellknorpels während einer mikrolaryngoskopischen Untersuchung des Kehlkopfs in Narkose.

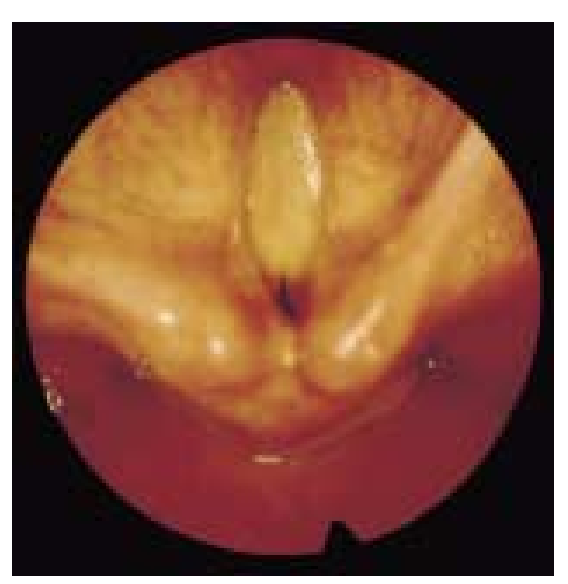

Abb. 5 Massive Ankylose im Cricoarytaenoidgelenk beidseits.

Wird eine ein- oder beidseitige Recurrenslähmung hingegen ohne vorangegangenes adäquates Trauma erstmals klinisch manifest, muss die Ätiologie der Parese umfassend geklärt werden. Dazu gehören neben nuklearmedizinischen und laborchemischen Untersuchungen der Schilddrüse insbesondere bildgebende Verfahren. Zum Ausschluss eines arrodierenden Prozesses sollte der gesamte Verlauf des Nervus recurrens von der Schädelbasis über die Halsweichteile bis zum Mediastinum durch computertomographische oder kernspintomographische Aufnahmen kontrolliert werden. Auch neurotrope Viren werden immer wieder als mögliche Auslöser 
10 -

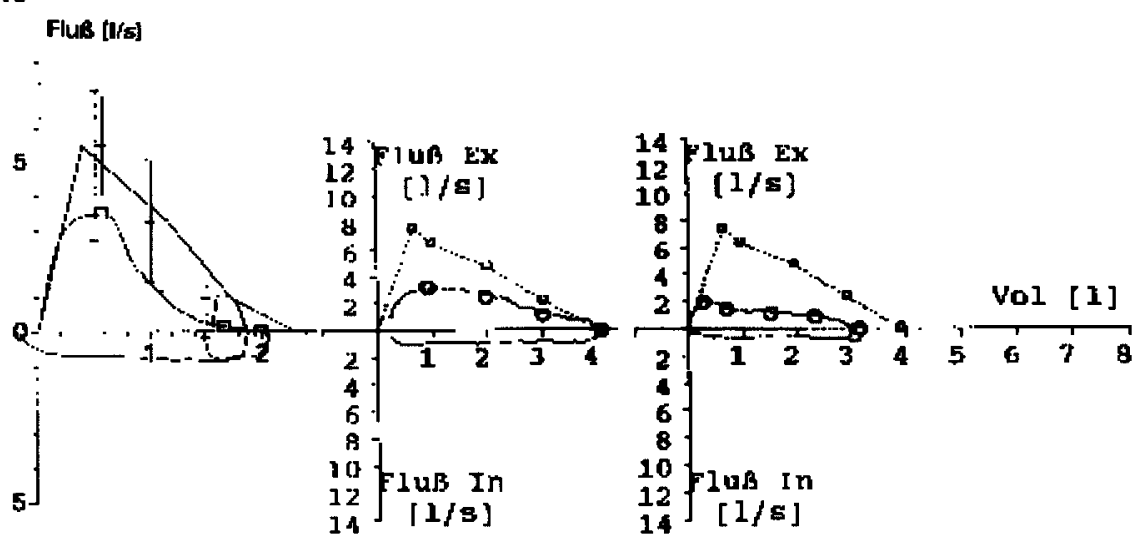

Abb. 6 Linke Bildseite normale Fluß-Volumen-Kurve. Oberer Teil von links nach rechts forcierte Exspiration nach maximaler Einatmung. Die Spitze des Dreiecks entspricht dem „peak expiratory flow“ (PEF). Unterer Teil von rechts nach links forcierte Inspiration nach maximaler Ausatmung. Der tiefste Punkt der Kurve entspricht dem „peak inspiratory flow" (PIF). Rechte Bildseite FlußVolumen-Kurve eines Patienten mit subglottischer Narbenstenose. Abflachung des Kurvenverlaufs mit Plateaubildung. einer Parese des Nervus recurrens angesprochen. Der endgültige Beweis dieser Hypothese steht jedoch noch aus, auch die Wirksamkeit einer virostatischen Therapie ist keinesfalls belegt. Aus unserer Sicht ist die routinemäßige serologische Testung auf Antikörper gegen neurotrope Viren daher entbehrlich, da die Ergebnisse klinisch ohne Konsequenz bleiben.

\section{Respiratorische Funktionsdiagnostik}

Unter physiologischen Bedingungen weisen die zentralen Atemwege des Menschen im Bereich der Trachea einen Querschnitt von etwa 300 bis $500 \mathrm{~mm}^{2}$ auf, im Bereich der Subglottis einen Querschnitt von etwa 200 bis $300 \mathrm{~mm}^{2}$ und im Bereich der Glottis 150 bis $200 \mathrm{~mm}^{2}$. Daher ist der Kehlkopf und speziell die Glottis bereits unter physiologischen Bedingungen stets der engste Teil der zentralen Atemwege $[17,18]$. Bei Lähmung der Stimmlippen in Adduktionsstellung vermindert sich der Winkel, den die beiden Stimmlippen in Respirationsstellung an der vorderen Kommissur beschreiben, von etwa $50^{\circ}$ beim Mann, bzw. $60^{\circ}$ bei der Frau auf etwa 5 bis $15^{\circ}$. Als Folge hiervon verengt sich der Atemweg auf einen Querschnitt von etwa 30 bis $60 \mathrm{~mm}^{2}$, also etwa auf ein Viertel des normalen Atemwegsquerschnitt im Bereich der ohnehin engen Glottis. Hieraus erklärt sich, warum eine beidseitige Rekurrenslähmung stets zu einer erheblichen respiratorischen Beeinträchtigung und häufig zu einem inspiratorischen Stridor führt. Einseitige Lähmungen sind hingegen praktisch nie atemrelevant. Die bisweilen subjektiv beklagte Luftnot resultiert hier zumeist durch den unvollständigen Glottisschluss, der insbesondere bei längerem Sprechen zu einem unkontrollierten Luftverlust und damit zu wenig ökonomischem Atemverbrauch führt (phonatorische Dyspnoe).

Nicht jede Stenosierung der Glottis ist korrekturbedürftig. Mitentscheidend sind die Entstehungsbedingungen der Stenose (akut/chronisch) und die daraus resultierende Adaptation der Atemmuskulatur an den erhöhten zentralen Atemwegswiderstand (Training) und speziell das Ausmaß, in dem eine gewohnte körperliche Belastung durch das unphysiologische Atemhindernis eingeschränkt wird.

Die Fluss-Volumen-Kurve ist der einfachste und zugleich aussagekräftigste Funktionstest zur Diagnose einer zentralen Atemwegsobstruktion. Stenosen der Glottis verändern die Fluss-Volumen-Kurve über den widerstandserhöhenden Ef- fekt der im Bereich hoher Stromstärken entstehenden Turbulenz, und in geringerem Maße auch über den Effekt der Querschnittsabnahme [19,20].

Im Gegensatz zur anatomisch fixierten Narbenstenose der Subglottis oder der Trachea (Abb. 6) ist die Atemwegsverengung bei beidseitiger Rekurrenslähmung durch eine druckpassive Weitstellung der Stimmlippen in der Ausatmung und eine Einwärtsbewegung (Medialisierung) über einen Sogeffekt (Bernoulli) während der Inspiration gekennzeichnet. Diesem Verhalten entspricht das charakteristische Flussprofil einer extremen inspiratorischen Abflachung mit einem oft weitgehend regelrechten normalen exspiratorischen Muster (Abb. 7).

Ein empirisch festgelegter Grenzwert für eine behandlungspflichtige Stenose findet sich nach eigenen Untersuchungen bei einer intratracheal gemessenen inspiratorischen Resistance von mehr als $2,5 \mathrm{kPa} \times \mathrm{s} / 1$. Letztlich wird aber die Entscheidung zur Operation ganz wesentlich durch die Frage beeinflusst, bis zu welcher körperlichen Belastung der Patient die Stenose respiratorisch kompensieren kann [21].

\section{Elektrophysiologische Diagnostik}

Die Frage nach der Ausheilungswahrscheinlichkeit einer Lähmung des Nervus recurrens ist für den betroffenen Patienten sehr wichtig, zumal bei Personen, die beruflich auf ihre Stimmfunktion angewiesen sind, gravierende soziale Folgen damit verbunden sein können. Aber auch die Einleitung und Planung einer logopädischen Therapie oder einer stimmrehabilitierenden operativen Maßnahme wird durch eine möglichst exakte Prognose wesentlich erleichtert. Besondere Erwartungen und Anforderungen sind im Falle der beidseitigen Rekurrensparese mit einer prognostischen Aussage verknüpft: wegen der ungewöhnlichen Kombination aus prinzipieller Reversibilität der Erkrankung und Irreversibilität (im Ralle einer chirurgischen Glottiserweiterung) bzw. Invasivität (im Falle einer Tracheotomie) der therapeutischen Maßnahme sollen einerseits diejenigen Fälle selektiert werden, bei denen keine funktionell ausreichende Wiederherstellung der Stimmlippenbeweglichkeit eintreten wird, um durch eine mikrolaryngoskopische Glottiserweiterung die Atmung zu normalisieren. Dies soll zudem zu einem möglichst frühen Zeitpunkt nach Eintritt der Schädigung geschehen, um dem Patienten möglichst die Tracheotomie zu ersparen. Andererseits gilt es 


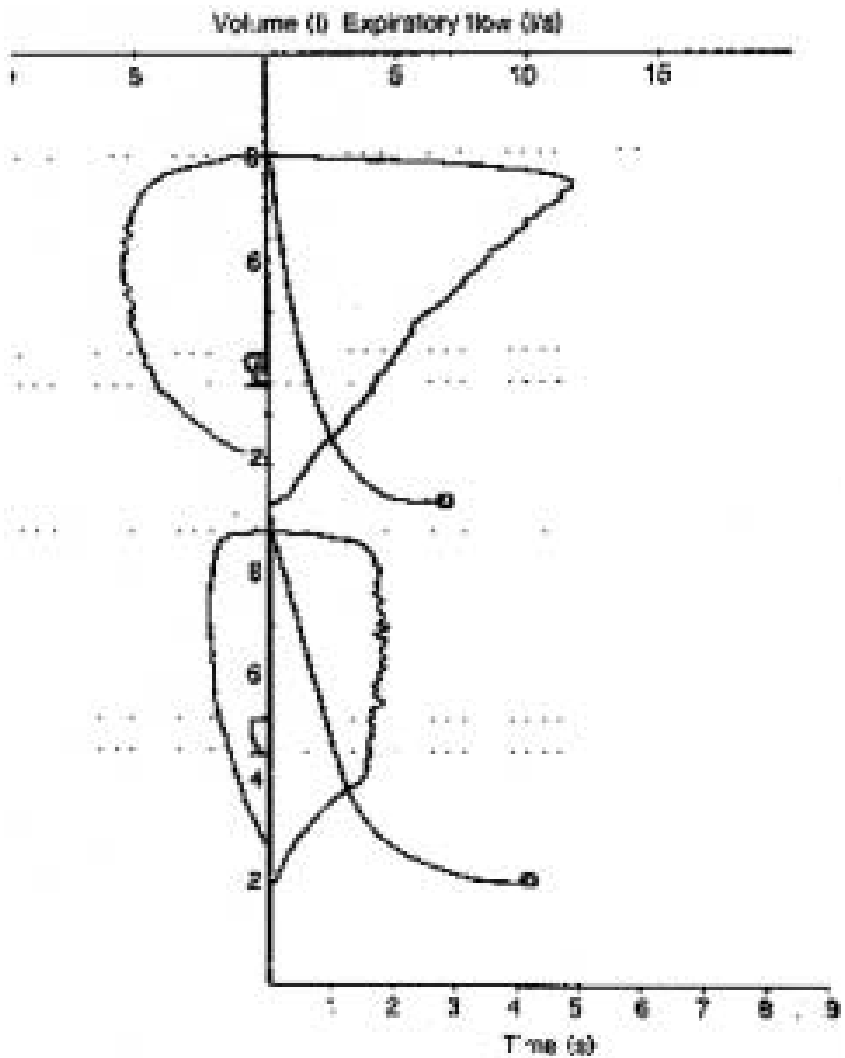

Abb. 7 Beidseitige Stimmlippenparese bei 3 Patientinnen nach Strumektomie, abnehmende Variabilität oder zunehmende Rigidität von links nach rechts. Während der Einatmung werden die „schlaffen“ Stimmlippen durch den Sogeffekt (Bernoulli) auf ihrer gesamten Länge in der Mittellinie aneinandergepresst (Adduktionsbewegung). Die so gebildete Stenose, die durch erhöhte Atemanstrengung nur noch verstärkt wird, erlaubt inspiratorische Flüsse von maximal 0,5 bis $0,8 \mathrm{l} / \mathrm{s}$. In der Ausatemphase hingegen wird die Glottis druckpassiv erweitert, und zwar umso mehr, je größer die Atemanstrengung. Damit kann ein normales exspiratorisches Flussprofil erzeugt werden (Kurvenbeispiel links). Eine Abflachung der exspiratorischen Kurve wie sie in rigiden zentralen Stenosen die Regel ist - entsteht bei beidseitigem Stimmlippenstillstand durch eine verminderte Atemanstrengung (Erschöpfung!) oder aber infolge einer sekundären Straffung der Stimmlippen (Atrophie) (mittleres und rechtes Kurvenbeispiel).

zu verhindern, dass Patienten einer definitiven Glottiserweiterung zugeführt werden, bei denen es auch ohne weitere Maßnahmen zu einer ausreichenden Erholung der Stimmlippenbeweglichkeit gekommen wäre. Vor diesem Hintergrund bietet allein die elektrophysiologische Diagnostik die Aussicht, hinreichend zuverlässige prognostische Informationen zu liefern.

Grundsätzlich basieren alle elektrophysiologischen Verfahren darauf, die Integrität der neuromuskulären Einheit über die Aufzeichnung von Aktionspotentialen der Kehlkopfmuskulatur zu überprüfen. Als wichtigste Potenzialformen unterscheidet man das motorische Willkürpotenzial, die pathologische Spontanaktivität und das Reinnervationspotential. In Ruhe ist der gesunde Muskel elektrisch inaktiv. Das Fehlen jeglicher elektrischer Aktivität bei dem Versuch einer Willkürbewegung wird hingegen als elektrische Stille bezeichnet.
Normale Willkürpotenziale der Kehlkopfmuskulatur sind dioder triphasisch und weisen extrem variable Amplituden auf (Abb. 8 und 9). Pathologische Spontanaktivitäten sind unwillkürliche Potentiale, die von einer einzelnen Muskelfaser rhythmisch generiert werden (Abb.10). Typisch ist für diese positiven scharfen Wellen vor allem der helle knackende Ton im Lautsprecher des Elektromyographen. Pathologische Spontanaktivität ist Ausdruck einer Nervendegeneration und tritt daher frühestens 10-14 Tage nach Schädigungsbeginn auf. Verwechslungen mit fortgeleiteten Aktionspotenzialen benachbarter Muskeln oder Endplattenpotentialen sind leicht möglich. Reinnervationspotentiale (Abb.11) sind plump verbreitert und mit mindestens 4 Phasendurchgängen polyphasisch. Sie kennzeichnen die Nervenerholung nach einer Wiederaussprossung der Axone.

Die elektrophysiologischen Befunde werden nach Seddon [22] in Neurapraxie, Axonotmesis und Neurotmesis eingeteilt. Diese Klassifikation beinhaltet eine prognostische Aussage: Die Neurapraxie heilt prinzipiell innerhalb von 8-12 Wochen

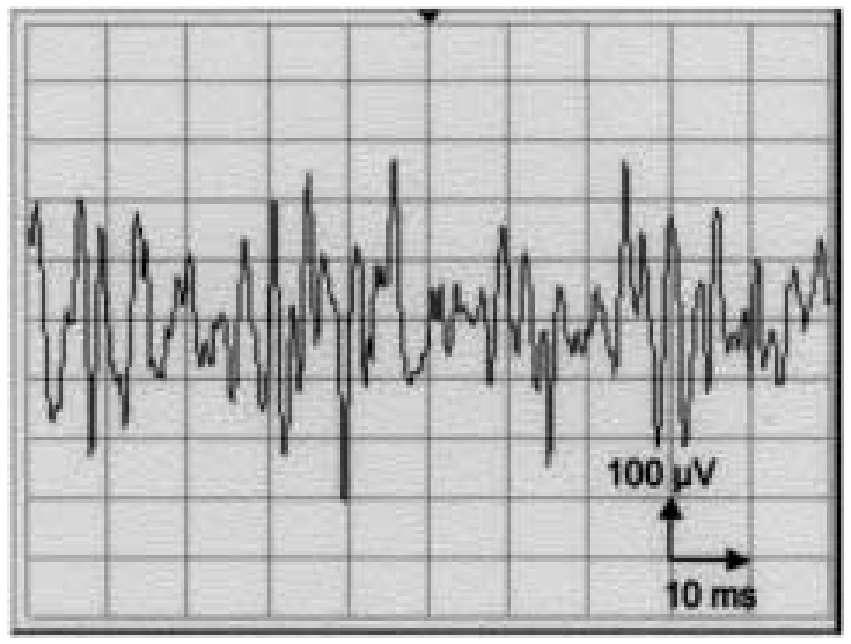

Abb. 8 Muskelaktionspotentiale des M. vocalis, gelichtete Interferenz.

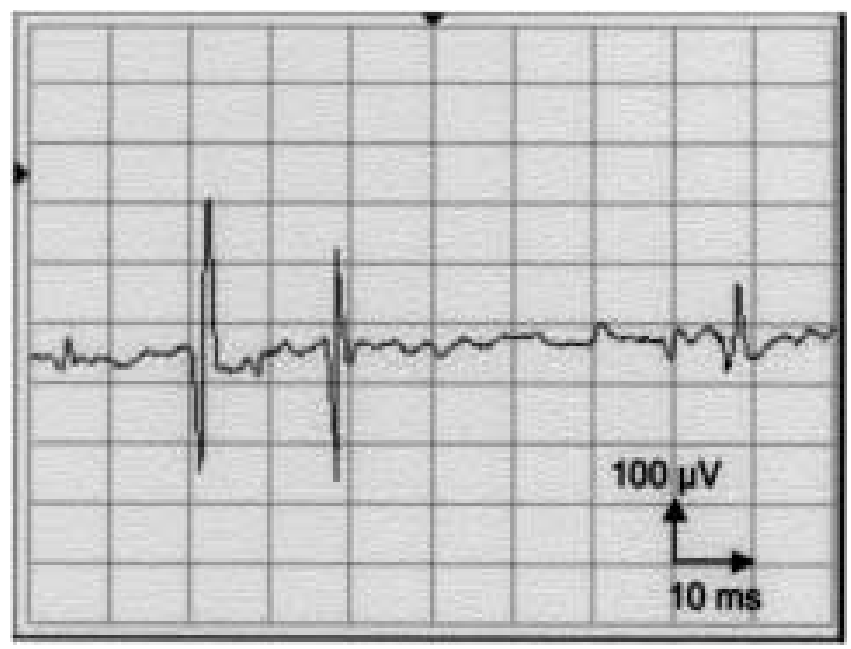

Abb.9 Muskelaktionspotential des M. vocalis, Einzelentladungen. 


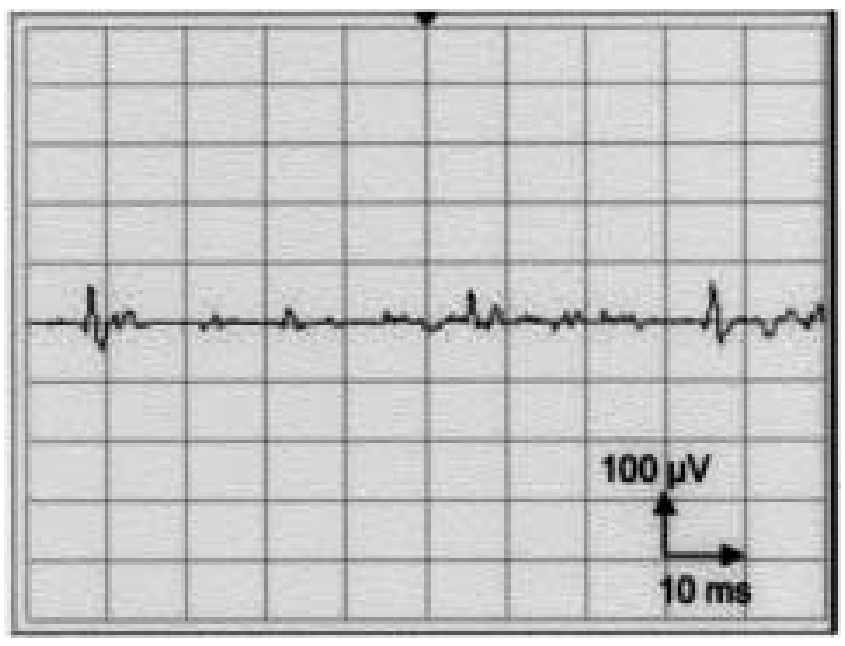

Abb.10 Spontane Fibrillationsaktivität in Ruhe, minimale Willküraktivität: Axonotmesis.

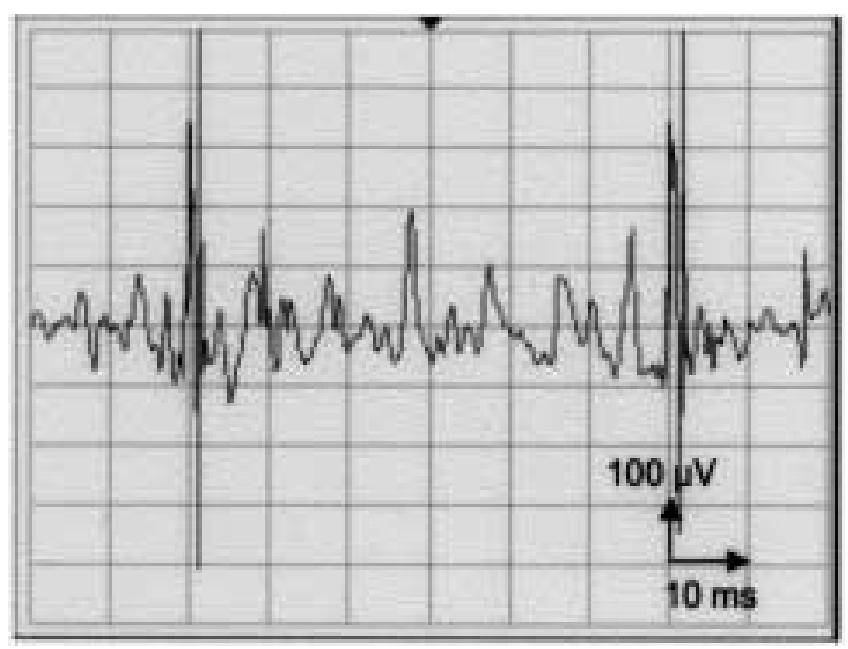

Abb.11 Polyphasische, verlängerte Potentiale mit Riesenamplituden: Reinnervation, 4 Monate nach Lähmungsbeginn.

folgenlos aus. Wie noch zu besprechen sein wird, gilt diese Aussage für die besondere Situation der Kehlkopfmuskulatur allerdings nur sehr eingeschränkt. Bei Vorliegen einer Axonotmesis ist die Wahrscheinlichkeit einer funktionell befriedigenden Ausheilung gering. Das Wiederaussprossen der Axone erfolgt ungerichtet und führt zu Synkinesien $[23,24]$. In der Folge kommt es zu einer simultanen Aktivierung der endolaryngealen Adduktoren. Endoskopisch imponiert ein straffer Stimmlippenstillstand, aber keine Beweglichkeit. In der simultanen 2-Kanal-Elektromyographie aus dem M. cricoarytaenoideus (Posticus) und dem M. thyroarytaenoideus medialis (Vocalis) beweisen Reinnervationspotenziale die grundsätzlich inaktive nervale Versorgung der Muskulatur. Dem betroffenen Patienten ist dieser Zustand gut durch das Bild des Automobils zu vermitteln, bei dem Gas und Bremse gleichzeitig betätigt werden: Obwohl alle Komponenten des Systems funktionsfähig sind, kommt es zu keiner sichtbaren Funktion.
Die Neurotmesis als Korrelat des vollständigen Nervenuntergangs führt zu einer vollständigen schlaffen Lähmung ohne Aussicht auf Erholung. Sie ist von der Axonotmesis nur durch den unterschiedlichen klinischen Verlauf zu unterscheiden. Im Grenzbereich sind die Übergänge zwischen einer weitgehenden Axonotmesis und einer Neurotmesis jedoch sicher fließend.

\section{Untersuchungstechnik}

Die Elektromyographie der inneren Kehlkopfmuskeln kann transoral und transkutan erfolgen. Bei der transoralen Ableitung wird unter endoskopischer Kontrolle eine dünne bipolare Elektrode aus Kupferdraht in die Stimmlippe eingestochen [25]. Für die transkutane Elektromyographie werden bipolare konzentrische Nadelelektroden in der Mittellinie der Membrana cricothyroidea knapp oberhalb des Cricoids eingestochen. Nach Überwinden der Membran wird die Nadel etwa $30^{\circ}-45^{\circ}$ nach oben und lateral in Richtung der zu untersuchenden Stimmlippe vorgeschoben. Die transkutane Technik ist technisch einfacher, kostengünstiger und wird von den meisten Patienten als weniger unangenehm empfunden. Die transorale Applikation erlaubt allerdings eine bessere Kontrolle über die korrekte Elektrodenlage. Die Gefahr der Verwechslung einer lähmungsbedingten elektrischen Stille mit einer Nullableitung wegen einer irrtümlichen Elektroden-Dislokation ist geringer. Da im Falle der beidseitigen Rekurrensparese diese Unterscheidung jedoch von erheblicher Bedeutung ist, und weitreichende Konsequenzen nach sich ziehen kann, favorisieren wir die transorale Technik zumindest bei der Diagnostik des beidseitigen Stimmlippenstillstands.

\section{Interpretation der Befunde}

Die Elektromyographie des Kehlkopfs ist eine dynamische Untersuchung, deren Interpretation nur im Kontext weiterer Befunde sinnvoll ist. Dynamisch bedeutet in diesem Zusammenhang, dass in die Bewertung zahlreiche weitere Faktoren einfließen, die nur aus dem laufenden Untersuchungsgang zu gewinnen sind. Anders als etwa bei der Elektrokardiographie ist die Beurteilung abgeleiteter Potentiale auf einem Papierstreifen allein nicht möglich. Wichtig ist insbesondere die akustische Information, die dem geschulten Ohr oft noch besser als die oszillografische Ableitung die Typisierung der Potentiale erlaubt. Auch das Verhalten der Potenziale bei Veränderungen der Elektrodenlage kann aufschlussreich sein, ebenso wie die Beobachtung des Patienten zur Erkennung von Artefakten, wie sie durch fortgeleitete Muskelaktivitäten bei ungenügend entspannten Patienten entstehen können.

Eine Auswertung eigener Daten von 540 Kehlkopf-Elektromyographien konnte nachweisen, dass die Elektromyographie des Kehlkopfs eine korrekte Vorhersage einer Defektheilung in $94,4 \%$ der Fälle erlaubt [26]. Es kann daher als gesichert gelten, dass der Nachweis von Degenerationszeichen in der Kehlkopf-Elektromyographie bereits zu einem frühen Zeitpunkt ein äußerst zuverlässiges Zeichen einer unvollständigen Nervenregeneration ist, die erst Monate später vollständig nachweisbar ist. Insbesondere für die beidseitige Rekurrensparese ist dies bedeutsam, weil im Fall einer ungünstigen Prognose die Entscheidung zu einer definitiven Glottiserweiterung zu einem wesentlich früheren Zeitpunkt erfolgen kann. Damit können minimal-invasive Verfahren der mikro- 
laryngoskopischen Glottiserweiterung zu einem vergleichsweise frühen Zeitpunkt indiziert werden. Wie am eigenen Krankengut gezeigt werden konnte [27], ist eine passagere Tracheotomie dadurch in nahezu allen Fällen vermeidbar. Die Gefahr der „voreiligen“ Operation einer Rekurrensparese, die sich später $\mathrm{zu}$ einem guten funktionellen Ergebnis erholt hätte, ist minimal. Gelingt der sichere Nachweis von Degenerationszeichen hingegen nicht, ist meist ein abwartendes Verhalten angezeigt. Eine Erholung ist dann prinzipiell möglich. Allerdings ist die Voraussage einer kompletten Ausheilung nur in einem sehr geringen Prozentsatz korrekt: So zeigt die bereits erwähnte Auswertung eigener Daten, dass die elektrophysiologische Diagnose Neurapraxie, definitionsgemäß eine vorübergehende Funktionsstörung mit hoher Wahrscheinlichkeit der vollständigen Erholung, für den N. recurrens lediglich in $12,8 \%$ der Fälle tatsächlich von einer restitutio ad integrum gefolgt wird. Die Faustregel „Neurapraxie = gute Prognose" scheint daher für den Bereich der Kehlkopfnerven nur sehr eingeschränkt zuzutreffen, wobei die Gründe dafür bislang größtenteils unklar sind.

Zusammengefasst lässt sich festhalten, dass der elektrophysiologische Nachweis von Degenerationszeichen zwar nahezu beweisend für die Entstehung einer Defektheilung ist, umgekehrt aber die Abwesenheit von Degenerationszeichen keineswegs zur Annahme einer völligen Funktionswiederkehr der gelähmten Stimmlippe berechtigt. Diese Einschränkung schmälert zwar die prognostische Aussagekraft der KehlkopfElektromyographie durchaus. Da aber das klinisch bedeutsamere Ereignis Defektheilung für einen großen Teil der Patienten akkurat vorhergesagt werden kann, hat die Kehlkopf-Elektromyographie dennoch einen bedeutsamen Platz im rationalen und effizienten Management der Recurrensparesen.

\section{Therapie}

Abhängig davon, ob es sich um einen einseitigen oder beidseitigen Stimmlippenstillstand handelt, liegen die Ziele der Behandlung diametral auseinander. Die einseitige Parese führt zu einer glottischen Schlussinsuffizienz und damit zu einer wenig tragfähigen und heiseren Stimme. Alle therapeutischen Maßnahmen stellen daher auf eine Verengung der Glottis ab. Die beidseitige Rekurrenslähmung führt hingegen zu einer Stenosierung des Atemwegs, hier ist die Erweiterung der Glottis indiziert.

\section{Die Therapie der unilateralen Parese}

Die große Mehrzahl der einseitigen Rekurrensparesen bedürfen keiner operativen Therapie. Zwar erscheint die Prognose prinzipiell recht ungünstig: So erreichten im eigenen Patientengut lediglich $8,1 \%$ aller Fälle eine folgenlose Ausheilung mit freier Beweglichkeit der zuvor paretischen Stimmlippe. Eine teilweise Wiederherstellung der Beweglichkeit wurde bei $46,9 \%$ der Fälle beobachtet. Tendenzielle Übereinstimmung mit den eigenen Daten zeigt eine Studie, die funktionelle Ergebnisse von Kehlkopflähmungen unterschiedlicher Ätiologie an einer großen Population untersuchte [28]. Eine wieder vollständig normalisierte Beweglichkeit wurde hier bei 21,8\% der Patienten gefunden, eine teilweise Wiederherstellung in $10,9 \%$. Alle übrigen Patienten wiesen eine persistierende, vollständige Lähmung auf. Allerdings kann auch eine deutlich eingeschränkte Funktion der inneren Kehlkopfmuskulatur gut kompensiert werden, bisweilen mit nur minimalen Folgen für die Stimmleistungsfähigkeit. So ist bekannt, dass die Phonation bei Patienten mit einer lediglich schwachen Adduktion oder Abduktion der Stimmlippen unbeeinträchtigt sein kann [29]. Eigene Ergebnisse sowie Daten aus der Literatur zeigen, dass 75-90\% der Patienten eine befriedigende bis nutzbare Stimmleistungsfähigkeit wiedererlangen, obwohl nur in $10-40 \%$ eine komplette Wiederherstellung der Stimmlippenbeweglichkeit beobachtet wurde.

Eine logopädische Therapie kann diese Kompensation erleichtern und beschleunigen und sollte großzügig indiziert werden. Wenn ein signifikanter glottischer Restspalt persistiert und der betroffene Patient subjektiv mit seiner Stimmleistungsfähigkeit unzufrieden ist, besteht die Indikation zur operativen Stimmverbesserung. Hierzu steht eine Vielzahl von Verfahren zur Verfügung, die alle darauf abzielen, die gelähmte Stimmlippe in eine mediane Position zu bringen, um somit der frei beweglichen Gegenseite ein festes Widerlager für einen kompletten Glottisschluss zu bieten. Die Thyreoplastik erreicht dies, indem ein Fenster im Schildknorpel geschaffen wird, durch das ein Implantat in den paraglottischen Raum eingesetzt wird (Abb.12) [30,31]. Der Eingriff erfolgt in örtlicher Betäubung, somit kann der optimale Sitz des Implantats angepasst werden, während der Patient phoniert. Bei der Augmentation wird auf mikrolaryngoskopischem Weg eine Substanz in die gelähmte Stimmlippe injiziert, die Volumenvermehrung bewirkt eine Verringerung des glottischen Restspalts (Abb.13). Ein Hautschnitt wird vermieden, dafür ist eine Vollnarkose erforderlich. Von den zahlreichen Substanzen, die zu diesem Zweck bereits verwendet wurden, haben sich insbesondere bovines Kollagen [32] und autologes Fett [33] bewährt. Beide Materialien werden allerdings im Laufe von 6-18 Monaten zum größten Teil resorbiert, so dass wiederholte Behandlungen notwendig werden. Neuerdings steht mit Bioplastique ${ }^{\mathrm{TM}}$ jedoch eine Substanz zur Verfügung, die eine dauerhafte und nebenwirkungsfreie Augmentation zu ermöglichen scheint [34].

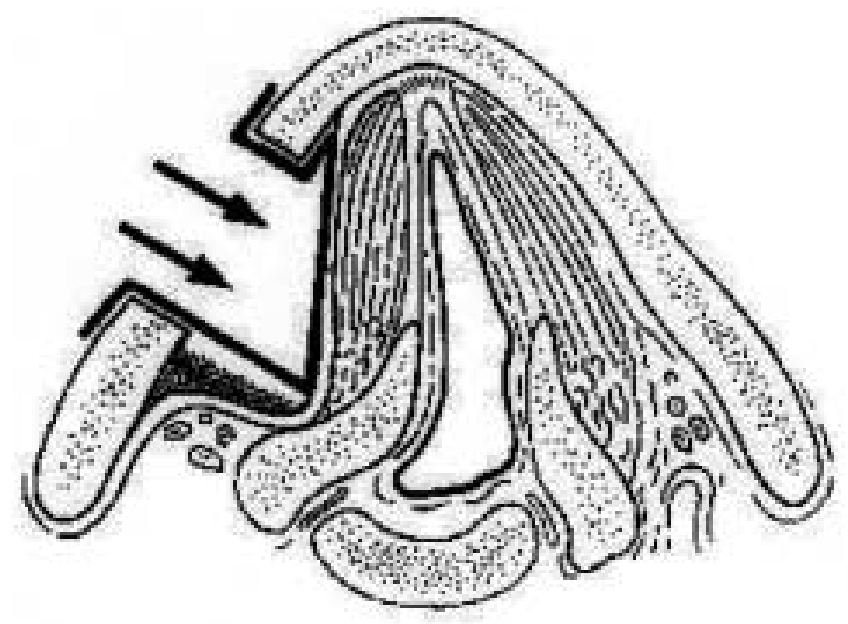

Abb.12 Schematische Darstellung einer Thyreoplastik: Titan-Implantat in situ (aus: G. Friedrich, Prophylaxe und Therapie von Stimmstörungen, in: G. Friedrich, W. Bigenzahn, P. Zorowka, Phoniatrie und Pädaudiologie, Hans Huber 2000, mit freundlicher Genehmigung von Prof. G. Friedrich, Graz). 


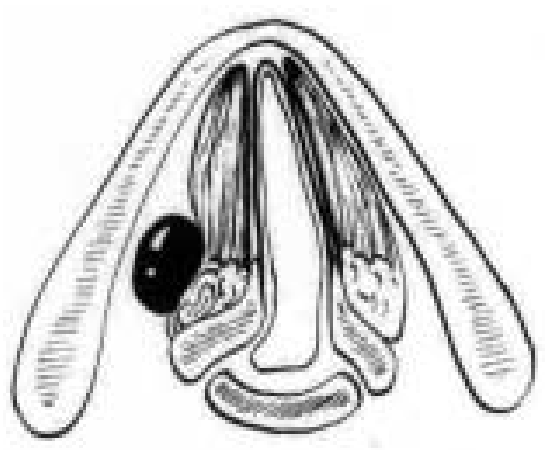

Abb. 13 Schematische Darstellung des Effekts einer Stimmlippen-Augmentation.

\section{Die Therapie der bilateralen Parese}

Die Behandlung der beidseitigen Rekurrenslähmung ist in aller Regel chirurgisch und verfolgt das vordringliche Ziel, die Atembehinderung der Patienten zu bessern [35].

In seltenen Fällen ist bei einer frisch aufgetretenen beidseitigen Rekurrenslähmung (etwa nach Schilddrüsenoperation) die Extubation unmöglich und daher unmittelbar die Tracheotomie erforderlich. In der Regel gelingt jedoch nach Gabe von Steroiden und nicht-steroidalen Antiphlogistika die Extubation innerhalb kurzer Zeit. Wenn die akute respiratorische Beeinträchtigung durch eine neu aufgetretene beidseitige Rekurrenslähmung durch Adaptation der Atemmuskulatur überwunden ist, können die meisten Patienten in Ruhe und bei geringer körperlicher Belastung ausreichend atmen. Für die Planung der weiteren Behandlung ist nun in erster Linie die Prognose der Nervenschädigung bedeutsam. Gelegentlich kann die Prognose einer Rekurrenslähmung nach der klinischen Grundsitutation abgeschätzt werden, etwa dann, wenn im Rahmen einer Thyreoidektomie wegen eines Schilddrüsenkarzinoms ein Nervus recurrens bewusst geopfert werden musste. Eine so klare Prognoseeinschätzung nur auf der Grundlage der klinischen Situation ist jedoch die Ausnahme. Die elektrophysiologische Diagnostik kann mit einiger Zuverlässigkeit zumindest die Defektheilung vorhersagen. Absolute Sicherheit lässt sich nur durch Zuwarten und Beobachten des Wiedereintritts/des Ausbleibens einer regelrechten Stimmlippenbeweglichkeit nach einem Zeitraum von 6 bis 9 Monaten gewinnen. Natürlich ist dabei zu bedenken, dass bei beidseitiger Lähmung die Prognose der Lähmung für die beiden betroffenen Seiten getrennt betrachtet werden muss. Die vollständige Erholung nur einer Seite ist ausreichend für eine Normalisierung der Atmung, chirurgische Maßnahmen erübrigen sich dann. Kommt es aber zu keiner ausreichenden Wiederherstellung der Stimmlippenbeweglichkeit, ergibt sich die Indikation zu glottiserweiternden Eingriffen einerseits aus dem Mangel an körperlicher Belastbarkeit, andererseits aus der potentiellen Gefährdung der Patienten durch sporadische Atemwegsentzündungen (grippale Effekte) mit nachfolgender Verschwellung der ohnehin sehr engen Glottis. Mit wenigen Ausnahmen ist erfahrungsgemäß bei einer bilateralen Rekurrenslähmung die Indikation zur glottiserweiternden Chirurgie gegeben, um die körperliche Belastbarkeit der Patienten jedenfalls teilweise wiederherzustellen und das Erstickungsrisiko im Rahmen von Atemwegsinfektionen zu vermindern.
Die bloße Beseitigung der Engstelle im Atemweg wäre chirurgisch einfach, sie würde aber durch einen nahezu vollständigen Stimmverlust erkauft werden. Die Herausforderung besteht darin, einen möglichst guten Kompromiss zwischen Respiration und Phonation zu finden. Trotz aller Fortschritte in der Kehlkopfchirurgie gilt immer noch, dass diese beiden Qualitäten zueinander in umgekehrt proportionalem Verhältnis stehen. Diese Zusammenhänge sollten mit dem Patienten vor einer geplanten Operation diskutiert werden, um gemeinsam die Ansprüche des Patienten an seine körperliche Leistungsfähigkeit und an seine Stimme einschätzen und einen für die individuelle Situation geeigneten Kompromiss anstreben zu können.

Die Tracheotomie ist das älteste und einfachste Verfahren zur Umgehung einer glottischen Atemwegsstenose. Sie ist sehr zuverlässig, komplikationsarm und kann bei Benutzung einer Sprechkanüle auch zu einer zufriedenstellenden stimmlichen Leistungsfähigkeit führen. Ein weiterer Vorteil ist, dass bei einer eventuellen Wiederkehr der Stimmlippenbeweglichkeit das Tracheostoma wieder verschlossen werden kann und der Kehlkopf funktionell wieder vollständig intakt ist. Allerdings werden die Behinderungen und Entstellung durch eine Tracheotomie von fast allen Patienten für so inakzeptabel gehalten, dass jedenfalls in der eigenen Praxis noch nie ein Patient, dem alle therapeutischen Optionen detailliert erläutert worden waren, sich für diesen Weg entschieden hat. Eine Tracheotomie als Dauerlösung der Atemwegsverengung bei beidseitiger Rekurrenslähmung muss daher in der Regel als inakzeptable Versorgung bezeichnet werden.

Lateralisierungsverfahren waren seit ihrer Einführung durch King 1939 weltweit die am häufigsten durchgeführten operativen Maßnahmen zur Erweiterung des Kehlkopfs bei beidseitiger Rekurrenslähmung. Sie ahmen dabei (einseitig) das natürliche Bild der Glottis in Inspirationsstellung nach (obwohl die statische Glottiserweiterung natürlich dem physiologischen Zustand des Kehlkopfs in keiner Weise entspricht). Im Laufe der Jahre wurden zahlreiche Modifikationen der ursprünglichen Operationstechnik angegeben. Alle zielen darauf hin, die glottischen Strukturen des Kehlkopfs (Stellknorpel und ligamentäre Glottis) nach lateral zu verlagern und so am Schildknorpel oder den bindegewebigen Strukturen des Halses zu fixieren, dass eine erneute Verengung der Glottis möglichst ausgeschlossen ist. Da beim intakten Larynx eine solche Lateralisation zu den physiologischen Bewegungsmöglichkeiten gehört, ist die statische Herstellung einer solchen Abduktion der glottischen Strukturen relativ einfach zu erzielen. Ein besonderer Vorteil dieses Verfahrens besteht darin, dass die schwingenden Strukturen der laterofixierten Stimmlippe anatomisch intakt und zumindest passiv für die Phonation erhalten bleiben.

Während diese Techniken primär über einen Zugang von außen durchgeführt wurden, gibt es seit jüngster Zeit auch die Möglichkeit der mikrolaryngoskopischen Lateralisation [36-39]. Dabei wird neben einer irreversiblen, dauerhaften Technik (in Verbindung mit einer Arytaenoidektomie) auch eine reversible Laterofixation (Lateralisation nur durch Naht) beschrieben. Allerdings liegen bislang keine ausreichenden Erkenntnisse darüber vor, ob eine temporäre Lateralisation tatsächlich wieder rückgängig gemacht werden kann, wenn ein vorübergehend gelähmter Nerv seine Funktion wieder 


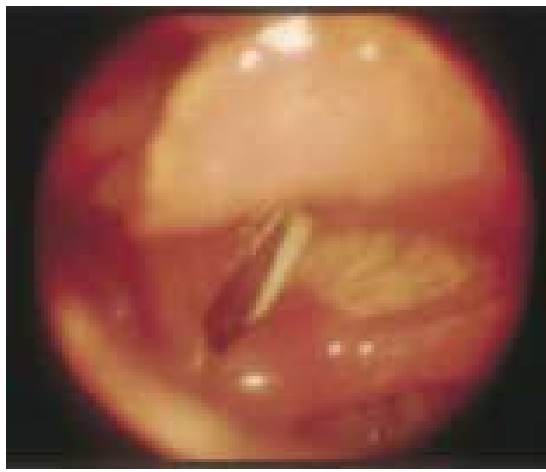

Abb. 14

Z.n. Laser-Arytaenoidektomie.

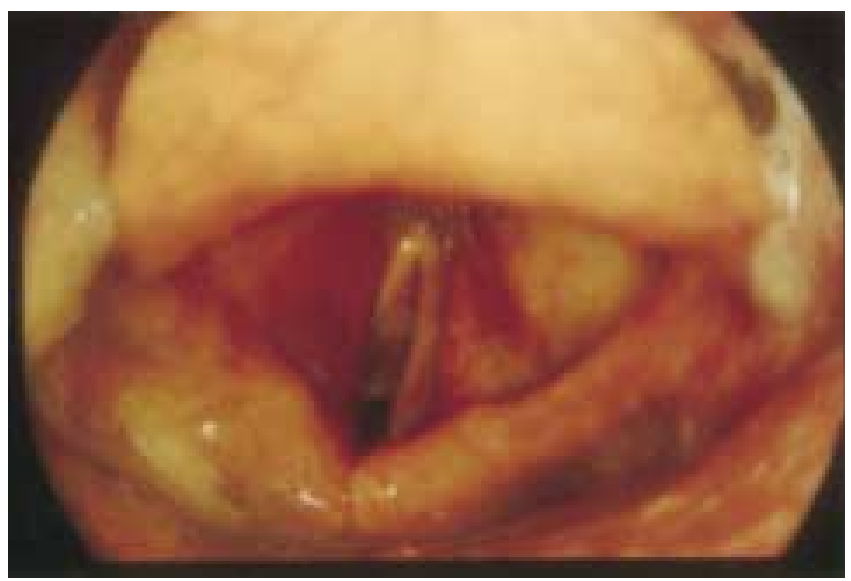

Abb.15 Z.n. posteriorer Chordektomie: Respirationsstellung.

findet, oder ob die länger bestehende Fixierung im CricoArytaenoidgelenk nicht doch zu einer bleibenden Fibrosierung der Gelenkkapsel oder zu einer Ankylose im Gelenk führt.

Endolaryngeale Resektionsverfahren: Die prinzipiell naheliegende Idee der Glottiserweiterung durch deren teilweise Resektion wurde erst mit den fortschreitenden Möglichkeiten der endoskopischen Chirurgie realisierbar. Kleinsasser nutzte die von ihm geschaffenen Möglichkeiten der mikrolaryngoskopischen Chirurgie und kombinierte die endolaryngeale Arytaenoidektomie mit einer submukösen Chordektomie [40]. Die Einführung des $\mathrm{CO}_{2}$-Lasers brachte eine wesentliche technische Erleichterung dieses Eingriffs, der nun sogar ohne temporäre Tracheotomie durchführbar wurde [41] (Abb. 14).

Seit dieser Zeit haben weitere endolaryngeale laserchirurgische Operationstechniken zur Glottiserweiterung klinische Anwendung gefunden: die Chordektomie [3,10,27,42-44] und die posteriore Chordektomie [45] (Abb.15). Die endolaryngealen Resektionsverfahren können heute als die weitest verbreitete Therapiemodalität zur chirurgischen Behandlung der bilateralen Rekurrensparese angesehen werden. Sie führen mit hoher Zuverlässigkeit $\mathrm{zu}$ einer ausreichenden Erweiterung des Atemwegs, erhalten aber zugleich in aller Regel eine befriedigende Stimmleistungsfähigkeit. Eine Aspiration ist hierbei nicht zu befürchten, und eine temporäre Tracheotomie ist in aller Regel nicht erforderlich (wenn der Patient postoperativ sehr sorgfältig überwacht werden kann und eine hochdosierte Steroidtherapie zur Prophylaxe von operationsbedingten Ödemen erfolgt). Der Eingriff eignet sich daher auch insbesondere zur Atemwegsrekanalisation bei beidseitiger Rekurrenslähmung durch fortgeschrittene Bronchialund Ösophaguskarzinome, er kann dann auch gleichzeitig mit der Implantation eines Tracheal-Stents erfolgen [11]. Der minimal-invasive mikrolaryngoskopische Eingriff belastet den Patienten nur wenig und ist technisch einfach genug, um auch außerhalb von spezialisierten Zentren durchgeführt zu werden.

Die ideale Lösung des Problems bestünde in einer gelungenen, funktionellen Reinnervation [46], da so die natürlichen Verhältnisse wiederhergestellt wären. Versuche einer einseitigen Reinnervation des N. laryngeus recurrens zeigten jedoch bisher nur teilweise elektromyographisch messbare Reinnervationspotentiale [47]. Hierdurch wird aber lediglich eine Tonisierung der endolaryngealen Muskulatur erreicht und nicht, wie erhofft, eine Wiederherstellung der Funktion. Eine funktionell vollständige Reinnervation wäre nur dann zu erwarten, wenn ad- und abduktorische Fasern des zentralen

Tab. 1 Die inneren Kehlkopfmuskeln: Übersicht der Funktion und Innervation

\begin{tabular}{|c|c|c|c|c|}
\hline anatomischer Name & klinischer Name & Funktion & Innervation & Besonderheit \\
\hline $\begin{array}{l}\text { M. cricoarytaenoideus } \\
\text { posterior }\end{array}$ & Posticus & $\begin{array}{l}\text { Abduktion der Stimmlippen, als } \\
\text { Antagonist Stimmlippenspanner }\end{array}$ & Nervus recurrens & einziger Öffner der Stimmritze \\
\hline $\begin{array}{l}\text { M. cricoarytaenoideus } \\
\text { lateralis }\end{array}$ & Lateralis & $\begin{array}{l}\text { wichtigster Adduktor der Stimm- } \\
\text { lippen. In Medianstellung auch } \\
\text { Stimmlippenspanner }\end{array}$ & Nervus recurrens & $\begin{array}{l}\text { dreht die Arytaenoidknorpel } \\
\text { einwärts }\end{array}$ \\
\hline M. interarytaenoideus & & $\begin{array}{l}\text { Adduktion der Stimmlippen ins- } \\
\text { besondere im Bereich der hinteren } \\
\text { Kommissur }\end{array}$ & Nervus recurrens & $\begin{array}{l}\text { überschreitet die Mittellinie und } \\
\text { wird von beiden } \mathrm{Nn} \text {. recurrentes } \\
\text { versorgt }\end{array}$ \\
\hline M. thyroarytaenoideus & $\begin{array}{l}\text { Vocalis } \\
\text { (medialer Anteil) }\end{array}$ & $\begin{array}{l}\text { spannt die adduzierten Stimm- } \\
\text { lippen }\end{array}$ & Nervus recurrens & $\begin{array}{l}\text { die medialen Anteile inserieren } \\
\text { direkt unterhalb der Stimmlippen } \\
\text { und beeinflussen so deren Vibra- } \\
\text { tionseigenschaften }\end{array}$ \\
\hline M. cricothyroideus & Anticus & $\begin{array}{l}\text { zusätzliche Spannung der Stimm- } \\
\text { lippen (nur bei sehr hohen Tönen) }\end{array}$ & $\begin{array}{l}\text { N. laryngeus } \\
\text { superior }\end{array}$ & $\begin{array}{l}\text { kein innerer Kehlkopfmuskel im } \\
\text { engeren Sinn. Einziger Kehlkopf- } \\
\text { muskel, der nicht vom Nervus } \\
\text { recurrens innerviert wird }\end{array}$ \\
\hline
\end{tabular}


Stumpfes des $\mathrm{N}$. laryngeus recurrens an die jeweiligen Neurone des peripheren Teiles Anschluss fänden. Da dies sehr unwahrscheinlich ist und die Leistungslimitierung durch die Stenose rein ventilatorisch bedingt ist [48], verspricht derzeit nur die mechanische, also operative Beseitigung oder Umgehung der Engstelle Besserung.

\section{Fazit für die Praxis}

Lähmungen des Nervus recurrens entstehen zumeist als Folge operativer Eingriffe, insbesondere an der Schilddrüse. Zur Planung der Therapie ist die Prognose der Lähmung von besonderer Bedeutung. Die Elektromyographie kann hier häufig eine wertvolle Hilfestellung leisten, sie dient darüber hinaus zur Abgrenzung gegenüber einer Stellknorpelankylose. Unilaterale Paresen führen $\mathrm{zu}$ einem Verlust an Stimmleistungsfähigkeit. Kommt es unter logopädischer Therapie zu keiner ausreichenden Kompensation besteht die Möglichkeit der operativen Stimmverbesserung mittels offener oder mikrolaryngoskopischer Verfahren. Bilaterale Schädigungen des Nervus recurrens führen stets zu einer erheblichen zentralen Atemwegsverengung, die in der Regel operativ korrigiert werden muss. Zur Behandlung stehen extralaryngeale als auch mikrolaryngoskopische Verfahren zur Verfügung, die zuverlässig eine ausreichende Atemwegserweiterung herbeiführen können, wobei der Verlust an Stimmleistungsfähigkeit tolerabel bleibt (Tab. 2 ).

\section{Literatur}

${ }^{1}$ Kelchner LN, Stemple JC, Gerdemann E, Le Borgne W, Adam S. Etiology, pathophysiology, treatment choices, and voice results for unilateral adductor vocal fold paralysis: a 3-year retrospective. J Voice 1999; 13: 592 - 601

${ }^{2}$ Remacle M, Lawson G, Mayne A, Jamart J. Subtotal carbon dioxide laser arytenoidectomy by endoscopic approach for treatment of bilateral cord immobility in adduction. Ann Otol Rhinol Laryngol 1996; 105: 438-445

${ }^{3}$ Eckel HE. Minimal-invasive Kehlkopfchirurgie zur Wiederherstellung des laryngealen Atemwegs bei beidseitiger Recurrenslähmung. Med Welt 1997; 48: 176-182

${ }^{4}$ Steinert M, Friedrich T, Keitel R, Sattler B, Schonfelder M. Indikation und operative Therapie von Schilddrüsenerkrankungen - Analyse von 725 operierten Patienten. Zentralbl Chir 1998; 123: $30-33$

${ }^{5}$ Hermann M, Keminger K, Kober F, Nekahm D. Risikofaktoren der Recurrensparese. Ein statistische Analyse von 7566 Strumaoperationen. Chirurg 1991; 62: $182-187$

${ }^{6}$ Jung H, Schlager B. Rekurrensparesen nach Strumektomien. Laryngorhinootologie 2000; 79: 297 - 303

${ }^{7}$ Thermann M, Feltkamp M, Elies W, Windhorst T. Recurrenslähmungen nach Schilddrüsenoperationen. Ursachen und Konsequenzen. Chirurg 1998; 69: 951 - 956

${ }^{8}$ Neumann HJ. Intraoperatives neurophysiologisches Monitoring (IONM) des Nervus recurrens und Mikrodissektion. Operationstechnisches Verfahren zur Risikominderung von Recurrensparesen. Laryngorhinootologie 2000; 79: 290-296

${ }^{9}$ Schilling MK, Seiler C, Schafer M, Buchler MW. Vermeidung der Rekurrensparese nach Schilddrüsenchirurgie - Versuch einer Metaanalyse. Ther Umsch 1999; 56: 396 - 399

${ }^{10}$ Eckel HE. Die laserchirurgische mikrolaryngoskopische Glottiserweiterung zur Behandlung der beidseitigen RecurrenspareseOperationstechnik und Ergebnisse. Laryngorhinootologie 1991; 70: $17-20$
${ }^{11}$ Wassermann K, Mathen F, Eckel HE. Malignant laryngotracheal obstruction: A way to treat serial stenoses of the upper airways. Ann Thorac Surg 2000; 70: 1197-1201

12 Kenn K, Schmitz M. „Vocal cord dysfunction“, eine wichtige Differentialdiagnose zum schweren und inplausiblen Asthma bronchiale. Pneumologie 1997; 51: 14-18

${ }^{13}$ Koufman JA, Walker FO, Ghazi MJ. The cricothyroid muscle does not influence vocal fold position in laryngeal paralysis. Laryngoscope 1995; 105: 368 - 372

${ }^{14}$ Paulsen FP, Rudert HH, Tillmann BN. New insights into the pathomechanism of postintubation arytenoid subluxation. Anesthesiology 1999; 91: 659-666

${ }^{15}$ Paulsen FP, Tillmann BN. Degenerative changes in the human cricoarytenoid joint. Arch Otolaryngol Head Neck Surg 1998; 124: $903-906$

${ }^{16}$ Thumfart WF. From larynx to vocal ability. New electro-physiological data. Acta Otolaryngol (Stockh) 1988; 105: 425 - 431

${ }^{17}$ Eckel HE, Sittel C. Morphometrische Untersuchungen der Glottisebene als Grundlage kehlkopferweiternder Operationsverfahren bei beidseitiger Rekurrenslähmung. Laryngorhinootologie 1994; 73: 417-422

${ }^{18}$ Eckel HE, Sittel C. Morphometry of the larynx in horizontal sections. Am J Otolaryngol 1995; 16: 40-48

${ }^{19}$ Wassermann K, Eckel HE. Funktionsdiagnostik zentraler Atemwegsstenosen. HNO 1999 Nov; 47: 947 - 956

${ }^{20}$ Wassermann K, Koch A, Warschow A, Mathen F, Muller-Ehmsen J, Eckel HE. Measuring in situ central airway resistance in patients with laryngotracheal stenosis. Larnygoscope 1999; 109: $1516-1520$

${ }^{21}$ Wassermann K, Gitt A, Weyde J, Eckel HE. Lung function changes and exercise-induced ventilatory responses to external resistive loads in normal subjects. Respiration 1995; 62: 177-184

22 Seddon H. Three types of nerve injury. Brain 1943; 66: 237-288

${ }^{23}$ Blitzer A, Jahn AF, Keidar A. Semon's law revisited: an electromyographic analysis of laryngeal synkinesis. Ann Otol Rhinol Laryngol 1996 Oct; 105: 764-769

${ }^{24}$ Sasaki CT, Horiuchi M, Ikari T, Kirchner JA. Vocal cord positioning by selective denervation. Old territory revisited. Ann Otol Rhinol Laryngol 1980; 89: 541 - 546

${ }^{25}$ Pototschnig C, Thumfart WF. Electromyographic evaluation of vocal cord disorders. Acta Otorhinolaryngol Belg 1997; 51: 99 104

${ }^{26}$ Sittel C, Stennert E, Thumfart WF, Dapunt U, Eckel HE. Prognostic value of laryngeal electromyography in vocal fold paralysis. Arch Otolaryngol 2001; 127: 155-160

${ }^{27}$ Eckel HE, Thumfart M, Wassermann K, Vossing M, Thumfart WF. Cordectomy versus arytenoidectomy in the management of bilateral vocal cord paralysis. Ann Otol Rhinol Laryngol 1994; 103: $852-857$

${ }^{28}$ Yamada M, Hirano M, Ohkubo $H$. Recurrent laryngeal nerve paralysis. A 10 -year review of 564 patients. Auris Nasus Larynx 1983; 10 Suppl: S1 - 15

${ }^{29}$ Crumley RL. Unilateral recurrent laryngeal nerve paralysis. J Voice 1994; 8: 79-83

${ }^{30}$ Friedrich G. Titanium vocal fold medializing implant: introducing a novel implant system for external vocal fold medialization. Ann Otol Rhinol Laryngol 1999; 108: 79-86

${ }^{31}$ Friedrich G. (External vocal cord medialization: functional outcome). Laryngorhinootologe 1998; 77: 18-26

${ }^{32}$ Remacle M, Marbaix E, Hamoir M, Bertrand B, van den Eeckhaut J. Correction of glottic insufficiency by collagen injection. Ann Otol Rhinol Laryngol 1990; 99: 438-444

${ }^{33}$ Shaw GY, Szewczyk MA, Searle J, Woodroof J. Autologous fat injection into the vocal folds: technical considerations and longterm follow-up. Laryngoscope 1997; 107: 177-186 
${ }^{34}$ Sittel C, Thumfart WF, Pototschnig C, Wittekindt C, Eckel HE. Textured polydimethylsiloxane elastomers in the human larynx: Safety and efficiency of use. J Biomed Mater Res 2000; 53: 646 650

${ }^{35}$ Eckel HE, Sittel C. Beidseitige Rekurrenslähmungen. HNO 2001; 49: $166-179$

${ }^{36}$ Jori J, Rovo L, Czigner J. Vocal cord laterofixation as early treatment for acute bilateral abductor paralysis after thyroid surgery. Eur Arch Otorhinolaryngol 1998; 255: 375-378

${ }^{37}$ Lichtenberger G, Toohill RJ. Technique of endo-extralaryngeal suture lateralization for bilateral abductor vocal cord paralysis. Laryngoscope 1997; 107: 1281 - 1283

${ }^{38}$ Lichtenberger G. Reversible immediate and definitive lateralization of paralyzed vocal cords. Eur Arch Otorhinolaryngol 1999; 256: $407-411$

${ }^{39}$ Rovo L, Jori J, Brzozka M, Czigner J. Airway complication after thyroid surgery: minimally invasive management of bilateral recurrent nerve injury. Laryngoscope 2000; 110: 140-144

${ }^{40}$ Kleinsasser O, Nolte E. Endolaryngeale Arytaenoidektomie und submuköse partielle Chordektomie bei bilateralen Stimmbandlähmungen. Bericht über 120 Fälle. Laryngorhinootologie 1981; 60: $397-397$

41 Ossoff RH, Duncavage JA, Shapshay SM, Krespi YP, Sisson GH. Endoscopic laser arytenoidectomy revisited. Ann Otol Rhinol Laryngol 1990; 99: 764-769

${ }^{42}$ Eckel HE. Minimal-invasive Kehlkopfchirurgie zur Wiederherstellung des laryngealen Atemwegs bei beidseitiger Rekurrenslähmung. Med Welt 1997; 48: 176-182

${ }^{43}$ Eckel HE, Thumfart M, Wassermann K, Vossing M, Thumfart WF. Cordectomy versus arytenoidectomy in the management of bilateral vocal cord paralysis. Ann Otol Rhinol Laryngol 1994; 103: $852-857$

${ }^{44}$ Eckel HE. Die laserchirurgische mikrolarygoskopische Glottiserweiterung zur Behandlung der beidseitigen Rekurrensparese. Operationstechnik und Ergebnisse. Laryngorhinootologie 1991; 70: $17-20$

45 Dennis DP, Kashima H. Carbon dioxide laser posterior cordectomy for treatment of bilateral vocal cord paralysis. Ann Otol Rhinol Laryngol 1989; 98: 930-934

${ }^{46}$ Tucker HM. Long-term results of nerve-muscle pedicle reinnervation for laryngeal paralysis. Ann Otol Rhinol Laryngol 1989 Sep; 98: 674-676

${ }^{47}$ Thumfart WF. From larynx to vocal ability. Acta Otolaryngol Stockh 1988; 105: 425-431

${ }^{48}$ Wassermann K, Gitt A, Weyde J, Eckel HE. Lung function changes and exercise-induced ventilatory responses to external resistive loads in normal subjects. Respiration 1995; 62: 177-184
PD Dr. med. Michael Thomas

Medizinische Klinik A

Pneumologie

Universitätsklinikum Münster

Albert-Schweitzer-Str. 33

48149 Münster

E-mail: mthomas@uni-muenster.de

Priv.-Doz. Dr. med. C. Sittel

Universitäts-HNO-Klinik des Saarlandes

Kirrberger Str.

66421 Homburg

E-mail: christian.sittel@uniklinik-saarland.de 